\title{
Time resolved in-situ synchrotron radiation diffraction investigation of grain-growth kinetics in magnetic nanocomposites
}

\section{J. Bednarčík ${ }^{1,2, *}$, R. Nicula ${ }^{1}$, V.D. Cojocaru ${ }^{3}$, M. Stir ${ }^{1}$ and E. Burkel ${ }^{1}$}

${ }^{1}$ Institute of Physics, Rostock University, August-Bebel-Str. 55, 18055 Rostock, Germany

${ }^{2}$ Institute of Physics, P.J. Safarik University, Park Angelinum 9, 04154 Košice, Slovakia

${ }^{3}$ University POLITEHNICA Bucharest, Spl. Independentei 313, 77206 Bucharest, Romania

*Contact author; e-mail: Jozef.Bednarcik@upjs.sk

Keywords: in-situ powder diffraction, grain-growth kinetics, magnetic nanocomposites

\begin{abstract}
Nanocrystalline powders with nominal composition $\mathrm{Co}_{42} \mathrm{Fe}_{22} \mathrm{Sm}_{22} \mathrm{Nb}_{7} \mathrm{~B}_{4} \mathrm{Cu}_{2} \mathrm{Ag}_{1}$ were synthesized by high-energy ball-milling. In the as-milled state, the alloy specimens consist of nanocrystalline $\alpha-(\mathrm{Fe}, \mathrm{Co})$. The formation of hard magnetic $\mathrm{Sm}_{2} \mathrm{Co}_{17}$ or $\mathrm{Sm}_{2} \mathrm{Fe}_{17}$ phases within the surrounding $(\mathrm{Fe}, \mathrm{Co})$ matrix during continuous heating was followed by insitu X-ray diffraction experiments performed at the HASYLAB/DESY synchrotron radiation facility in Hamburg, Germany. The hard magnetic $\mathrm{Sm}_{2} \mathrm{Co}_{17}$ nanocrystalline phase forms at temperatures near to $800^{\circ} \mathrm{C}$. The temperature evolution of the grain-size distribution was obtained from the integral breadth double-Voigt analysis of the broadening of the (Fe,Co)matrix X-ray diffraction lines. The kinetics of microstructure coarsening seems to be influenced by grain boundary pinning (drag) effects correlated with the formation of a two-phase microstructure.
\end{abstract}

\section{Introduction}

Increasing efforts were recently devoted to the further development of exchange coupled nanocomposite magnets [1-5]. The exchange coupling between hard $(\mathrm{NdDy})_{2}(\mathrm{FeCo})_{14} \mathrm{~B}$ and soft-magnetic $\left.\alpha-(\mathrm{FeCo}), \mathrm{Fe}_{3} \mathrm{~B}\right)$ constituent phases was shown to improve the hard magnetic properties of nanocomposite permanent magnets prepared by melt-spinning and subsequent annealing [4,5]. We report on a first attempt to synthesize exchange coupled hard magnets by high-energy mechanical alloying of elemental powders, followed by the in-situ formation of magnetic nanocomposites during continuous heating. The formation of hard magnetic $\mathrm{Sm}_{2} \mathrm{Co}_{17}$ or $\mathrm{Sm}_{2} \mathrm{Fe}_{17}$ phases within the initial nanocrystalline $\alpha-(\mathrm{FeCo})$ matrix during heating was followed by in-situ X-ray diffraction experiments performed at the HASYLAB/DESY synchrotron radiation facility (Hamburg, Germany). 


\section{Experimental}

The powder samples with nominal composition of $\mathrm{Co}_{42} \mathrm{Fe}_{22} \mathrm{Sm}_{22} \mathrm{Nb}_{7} \mathrm{~B}_{4} \mathrm{Cu}_{2} \mathrm{Ag}_{1}$ (at.\%) were prepared from high purity $(>99.8 \%)$ powder elements. The corresponding alloy was obtained by means of mechanical alloying using a high-energy planetary ball mill RETSCH PM400. The powder mixture was wet-milled in hexane for 90 hours. Stainless steel vials and balls were used. Mechanical alloying was performed at a ball-to-powder (BPR) mass ratio of 18:1 with an angular speed of 250 rotations/minute (rpm). The temperature stability of the mechanically-alloyed specimens was investigated by in-situ angular-dispersive X-ray diffraction using the high-resolution diffractometer at the B2 beamline in HASYLAB/DESY. The synchrotron radiation wavelength was set to $\lambda=0.7095 \AA$. Heating experiments were performed in-situ, using an OBI image plate detector and a STOE furnace [6].

\section{Results and discussion}

The synchrotron radiation diffraction patterns collected between $300-900^{\circ} \mathrm{C}$ during constantrate heating are illustrated as a two-dimensional diffracted intensity map in Figure 1 . The Xray diffraction patterns were indexed to the body centered cubic bcc $(\mathrm{Fe}, \mathrm{Co})$ phase. Rather weak peaks were identified as belonging to the $\mathrm{Sm}_{2} \mathrm{Co}_{17}$ hard-magnetic nanocrystalline phase, at temperatures slightly exceeding $800^{\circ} \mathrm{C}$ (not shown). This indicates that the longrange diffusion of the large atomic radius $\mathrm{Sm}$ atoms, needed for the nucleation of the hard magnetic phase, is only triggered by relatively high temperatures. This behaviour may further be improved by adjusting the nominal composition of the starting alloy powders and by pressure application during specimen heating.

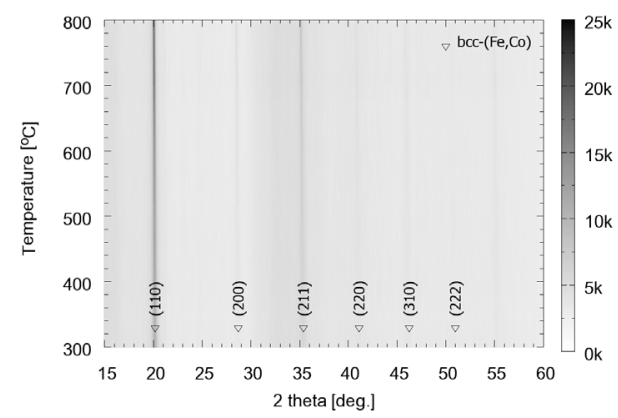

Figure 1. Synchrotron radiation powder diffraction patterns collected during constant-rate heating of the powder sample milled for 90 hours.

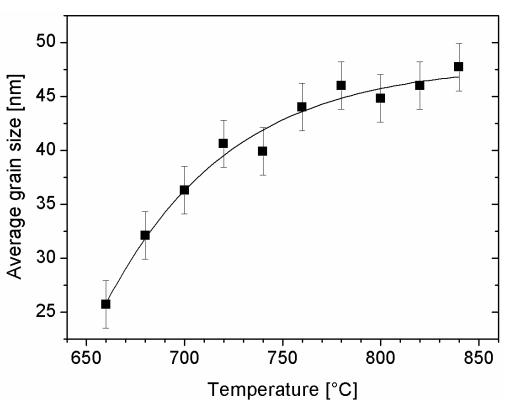

Figure 2. Temperature evolution of the average grain size for the bcc-(Fe, $\mathrm{Co})$ main phase of the CoFeSmNbBCuAg alloy.

In order to describe the microstructural evolution of the ( $\mathrm{Fe}, \mathrm{Co})$ matrix with temperature, after the full-pattern decomposition of the XRD patterns using PEAKFIT ${ }^{\mathcal{O}}$ software [7], the (110) and (220) diffraction lines of the bcc-(Fe,Co) nanocrystalline phase were used to infer the temperature-resolved volume-weighted average grain size (Figure 2), and grain-size distribution functions (Figure 3$)$ for the bcc $(\mathrm{Fe}, \mathrm{Co})$ phase. We used the double-Voigt line- 
profile analysis (LPA) method [8]. The resulting grain-size distributions were least-squares fitted to both log-normal and gamma distribution functions, respectively. Irrespective of temperature, the experimental grain-size distributions are better described by gamma distribution functions. The resulting area-normalized gamma distributions are shown in Figure 3. Cumulative grain size distributions were as well retrieved (Figure 4), yielding the fraction of grains having sizes below a certain arbitrary value. For example, one may readily conclude that while at $660^{\circ} \mathrm{C}$ about $82 \%$ of the grains are less than $40 \mathrm{~nm}$ in size, this fraction drops down to only $49 \%$ at $840^{\circ} \mathrm{C}$.

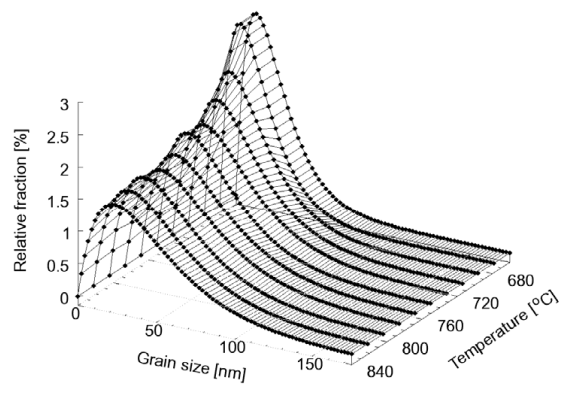

Figure 3. The temperature evolution of the area normalized grain-size distributions for as-milled sample.

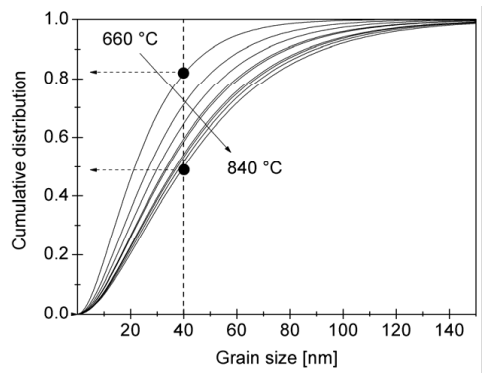

Figure 4. Cumulative distribution functions describing the changes of the microstructure during constant-rate heating (annealing).

The temperature- / time- dependence (coarsening kinetics) of the alloy microstructure may be followed in detail via the evolution of predefined crystallite-size populations (fractions) of the constituent grains. In-situ synchrotron radiation X-ray diffraction experiments therefore provide at specimen-relevant scales an accurate statistical description of the temperaturetime-dependent evolution of the microstructure. Information on nanocrystallization and grain-growth is furthermore essential to the guidance of sintering experiments; for instance, sintering rates were shown to vary significantly with the grain-size distribution widths [9].

\section{Concluding remarks}

Preliminary attempts were undertaken to produce exchange coupled nanocomposite magnets based on the $\mathrm{Sm}_{2} \mathrm{Co}_{17}$ hard magnetic and the bcc matrix (Fe, Co) soft magnetic phases. The synthesis procedure relies on the wet ball-milling of high-purity elemental powders followed by in-situ nanocomposite formation during subsequent heating. In-situ synchrotron radiation diffraction experiments show that the hard magnetic $\mathrm{Sm}_{2} \mathrm{Co}_{17}$ phase forms at temperatures immediately above $800^{\circ} \mathrm{C}$. Further studies will mainly aim to increase the fraction of the hard magnetic phase and the significant reduction of its nucleation temperature by increasing the Sm content of the starting alloy powders and by using isothermal annealing procedures at ambient and non-ambient pressure conditions. The necessary long-range diffusion of Sm and perhaps also $\mathrm{Nb}$ atoms will thus be supported. Moreover, as shown in Figure 2, the average crystallite size of the $(\mathrm{Fe}, \mathrm{Co})$ phase almost ceases to increase at temperatures approaching the nucleation onset of the $\mathrm{Sm}_{2} \mathrm{Co}_{17}$ phase (above $770^{\circ} \mathrm{C}$ ), clearly due to the formation of a 
two-phase microstructure and to Co atoms increasingly contributing to the new phase development within this temperature range. The expected reduction of the onset temperature for the nucleation of the hard magnetic phase (down to about $650^{\circ} \mathrm{C}$ ) by the above mentioned measures, should therefore also result in finer grain sizes of the nanocomposite (Fig. 4) and thus in improved hard magnetic properties through enhanced hard - soft exchange coupling.

\section{References}

1. Manaf, A., Buckley, R.A. \& Davies, H.A., 1993, J. Magn. Magn. Mater., 128, 302.

2. Withanawasam, L., Murphy, A.S., Hadjipanayis, G.C. \& Krause, R.F., 1994, J. Appl. Phys., 76, 7065.

3. Biswas, K., Ram, S., Roth, S., Schultz, L. \& Eckert, J., 2006, J. Mater. Sci., 41, 3445.

4. Zhang, W. \& Inoue, A., 2000, J. Appl. Phys., 87, 6122.

5. W. Zhang, W. \& Inoue, A., 2002, Appl. Phys. Lett., 80, 1610.

6. Knapp, M., Bähtz, C., Ehrenberg, H. \& Fuess, H., 2004, J. Sync. Rad., 11, 328-334.

7. PeakFit for Windows User's Manual, SPSS Science, Inc., ISBN 1-56827-183-2.

8. Balzar, D. \& Ledbetter, H., 1993, J. Appl. Cryst., 26, 97.

9. Chappell, J.S., Ring, T.A. \& Birchall, J.D., 1986, J. Appl. Phys., 60, 383.

Acknowledgements. Thanks are due to Dr. C. Bähtz and Dr. M. Knapp for their kind help with experiments at the B2 HASYLAB beamline. This work was financially supported by the Marie Curie Programme of the European Community (HPMD-CT-2001-00089) and by the Slovak grant agency APVV (APVT-20-008404). 